\title{
Neural Network Based on Model Reference Using for Robot Arm Identification and Control
}

\author{
Rafid A. Khalil \\ Mechatronics Engineering \\ Mosul University, Iraq \\ rafidamori@yahoo.com
}

\author{
Rakan Kh. Antar \\ Technical College /Mosul \\ Foundation of Technical Education, Iraq \\ rakanpe@yahoo.com
}

Abstract. In this work, neural network control theory is applied to identify and control the robot arm with two links conformed by two equations of second order which alternate their operation simultaneous. A neural network is trained to learn the robot arm in the dynamic behavior. The simulation results of the neural network controller based on model reference that used to identify and control the robot arm give very close results.

Keywords: Neural network controller, model reference, plant identification, robot arm.

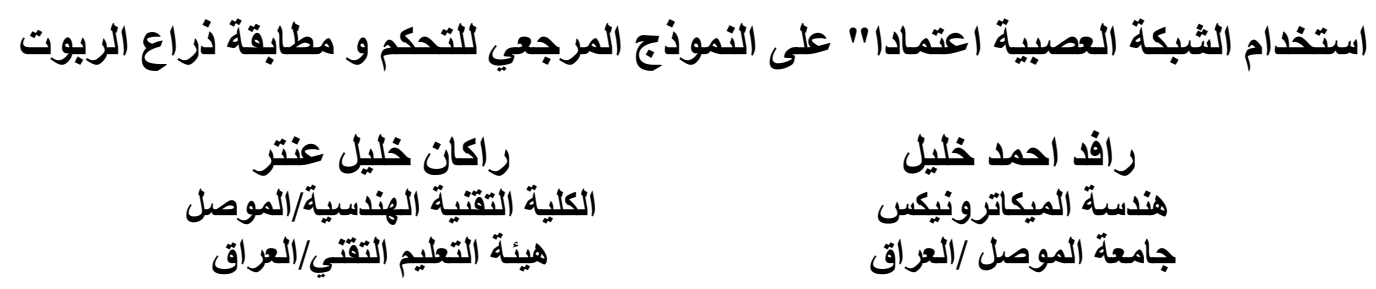

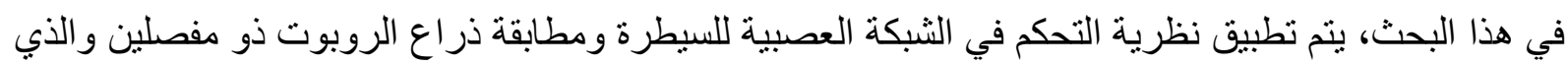

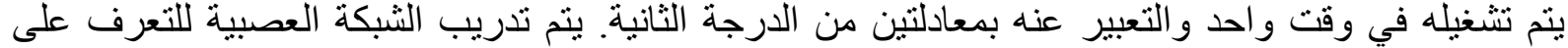

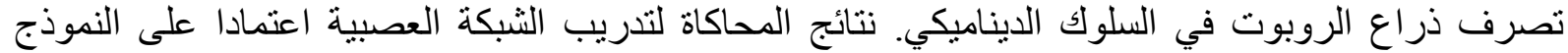

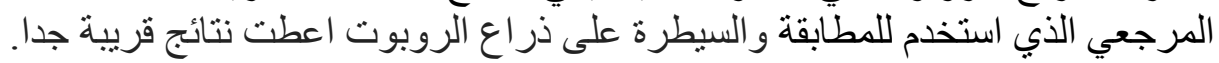




\section{Introduction}

Robotic manipulator has to face different suspicions in their dynamics, such as payload parameter, friction, and disturbance. It is complex to set up a suitable mathematical model to design a control system model. Therefore, the intelligent controllers can attenuate the structured parametric uncertainty and unstructured disturbance effects using their powerful learning ability. The identification of mathematical models using neural networks and fuzzy logic systems enables to recognize unknown parameters and adjust the mathematical model to the real object. Neural networks show the ability to obtain successful results in system identification and control [1, 2]. Neural networks have proved to be powerful and popular tools for learning motor control of robots with many degrees of freedom. It is well known that the demand for increased productivity by robots can be met by the use of lighter robots operating at high speed and consuming less energy, which may lead to a reduction in the stiffness of the manipulator structure. This would result in an increase in robot deflection and poor performance due to the effect of mechanical vibration in the links and bring difficulties for control [3]. In contrast, a robot system is a highly nonlinear and heavily coupled mechanical system. The mathematical model of such system usually consists of a set of nonlinear differential equations derived by using some form of approximation and simulation [3].

A robot control system should be designed and come up with a control scheme that will enable the proposed robotic system to perform the required tasks with a high degree of precision, accuracy and reliability particularly under various conditions involving the robot's interaction with its environment. Also the system has to be robust, stable and effectively capable to accomplish the prescribed tasks even in the presence of disturbances, parametric changes, uncertainties and varied operating conditions [4].

In this paper, a neural network (NN) controller based on the reference model technique applied on a robot arm with two links is achieved as shown in Fig.1. It is trained and designed to identify the robot arm model and the identification output signal compared with the actual robot arm output. This comparison is the input of the NN controller. This NN controller is designed in such a way that makes the robot arm output, which is angle of the arm, to follow the output of a reference model. Another neural network will be trained in order to operate as a robot arm's controller, taking into consideration the behavior of the reference model. The system is trained so that the two links are moved alternate [5].

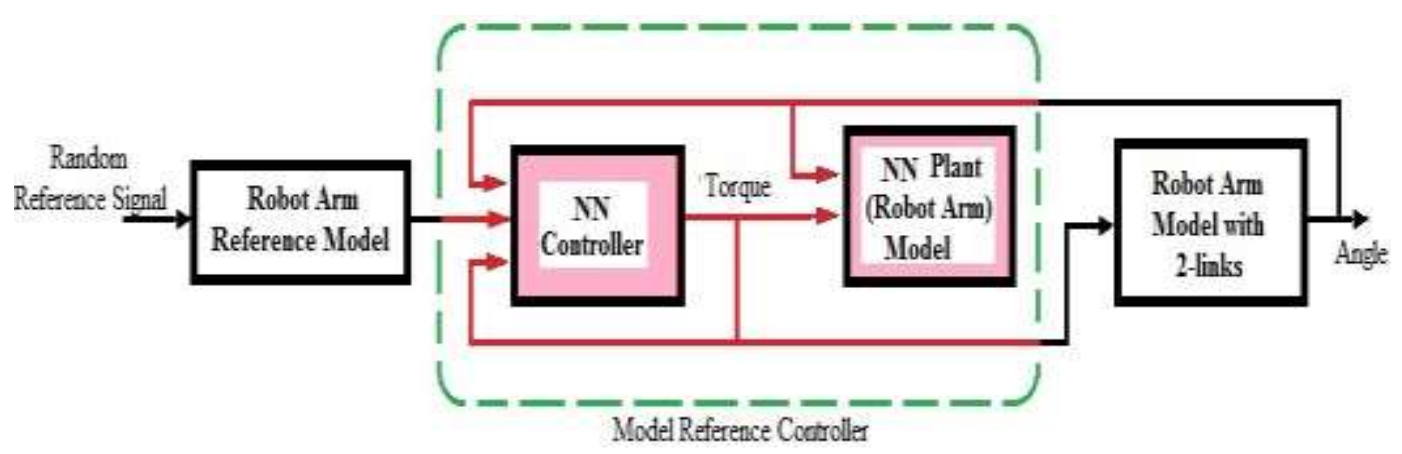

Fig.1 Proposed robot arm system. 


\section{The Robot Arm Description}

The robot used in this implementation is a two link arm with two rotational degrees of freedom restricted to movement in a plane. Many studies have used a manipulandum to restrict a person to move their arm in a plane, providing data for comparison between the movement of the robot and actual human movement [6]. Robot arm's equations with two links are given below. Equations (1) and (2) represent the scalar equations of a manipulator of a two-link robot arm, respectively [7]:

$$
\begin{aligned}
& M_{\theta 1} \ddot{\theta}_{1}+F_{d 1} \dot{\theta}_{1}+G_{1}(\theta)=\tau_{1} \\
& M_{\theta 2} \ddot{\theta}_{2}+F_{d 2} \dot{\theta}_{2}+G_{2}(\theta)=\tau_{2}
\end{aligned}
$$

Where $\theta$ denotes the link position, $\mathrm{M}_{\theta}$ is the inertia matrix, $G(\theta)$ represents the gravity vector, $F_{d}$ is a diagonal matrix representing the friction term, and $\tau$ is the input torque applied to the links. The two-links robot arm case is shown in Fig.2. For the simplest case of a onelink robot arm, we have the scalar equation:

$\ddot{\theta}_{1}+a_{1} \dot{\theta}_{1}+b_{1} * \sin \left(\theta_{2}\right)=c_{1}$

$\ddot{\theta}_{2}+a_{2} \dot{\theta}_{2}+b_{2} * \sin \left(\theta_{1}\right)=c_{2}$

where $a=\frac{F_{d}}{M_{\theta}}, b=\frac{G(\theta)}{M_{\theta}}$ and $c=\frac{\tau}{M_{\theta}}$

In this paper, equations (3) and (4) represented as:

$\ddot{\theta}_{1}+3.7 \dot{\theta}_{1}+13 \sin \left(\theta_{1}\right)=c_{1}$
$\ddot{\theta}_{2}+6 \dot{\theta}_{2}+13 \sin \left(\theta_{2}\right)=c_{2}$

The system was sampled at intervals of 0.05 seconds. The NN plant model is includes two delayed values of torque $(\mathrm{m}=2)$ and two delayed values of arm position $(n=2)$ as input to the network, and 10 neurons are used in the hidden layer (4-10-1 network). The objective of the control system is to make the arm track the reference model below:

$$
\begin{aligned}
& \ddot{y}_{1}+4 \dot{y}_{1}+5 y_{1}=5 u_{1} \\
& \ddot{y}_{2}+3.5 \dot{y}_{2}+4 y_{2}=5 u_{2}
\end{aligned}
$$

Where $y$ is the output of the reference model and $u$ is the input reference signal. For the controller network, it is used a (5-13-1 network) architecture. The inputs to the controller consisted of two delayed reference inputs, two delayed plant outputs, and one delayed controller output. The controller is trained using a BFGS quasi-Newton algorithm, with dynamic back-propagation used to calculate the gradients.

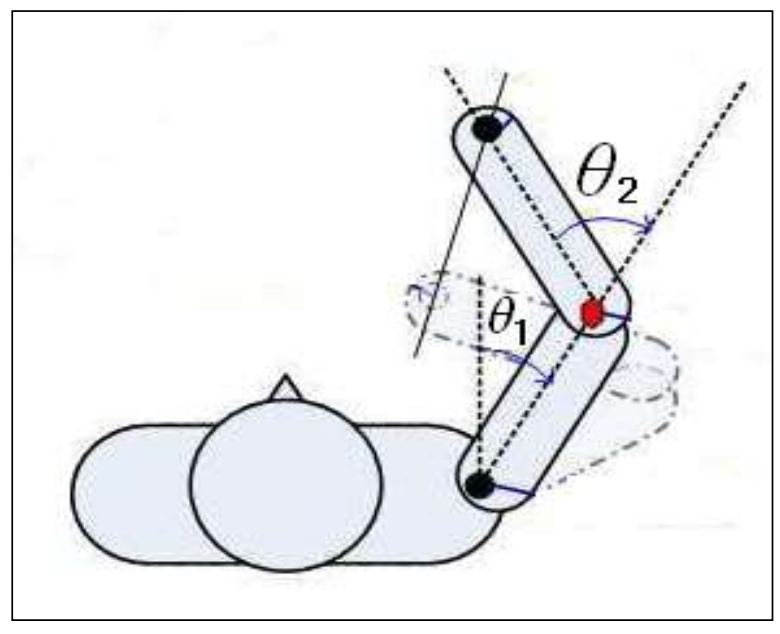

Fig. 2 Two Links Robot 


\section{Model Reference Design}

The robot arm reference model is chosen in order to make the neural network acts as a controller so to drive the robot arm to behave as the reference model. There are two neural networks: the plant identification one and the other realize the controller function. Neural Networks Toolbox offers the model reference controller strategy. It is very important to note the necessity of identifying the plant, first, before proceeding with the neural controller training.

To identify the plant, a Levenberg- Marquandt (trainlm) technique is used. Performance behavior of the identification plant process is measured through the mean square error (MSE) index as shown in Fig.3. The reported value for that index is $1.83751 \mathrm{e}-11$, which is good enough for all practical purposes where the Matlab stipulated goal for the performance index is zero. Plant identification process, training the neural network plant, validation and test data for model reference control results are illustrated in Fig.4 and Fig.5, respectively. After plant training was completed, training model reference control results are shown in Fig.6. The output does not follow the input exactly, due to the inertia and time constants of the plant (robot arm). To proceed with the training controller, Matlab uses a training technique named trinbfgc, which reported the performance shown in Fig.7.

Figure 8 explains the plant response for input and output of $\mathrm{NN}$ model reference control. After that the controller block is applied to the plant as shown in Fig.9. The internal structure of the model reference controller illustrated in Fig.10 is used as the plant and reference models.

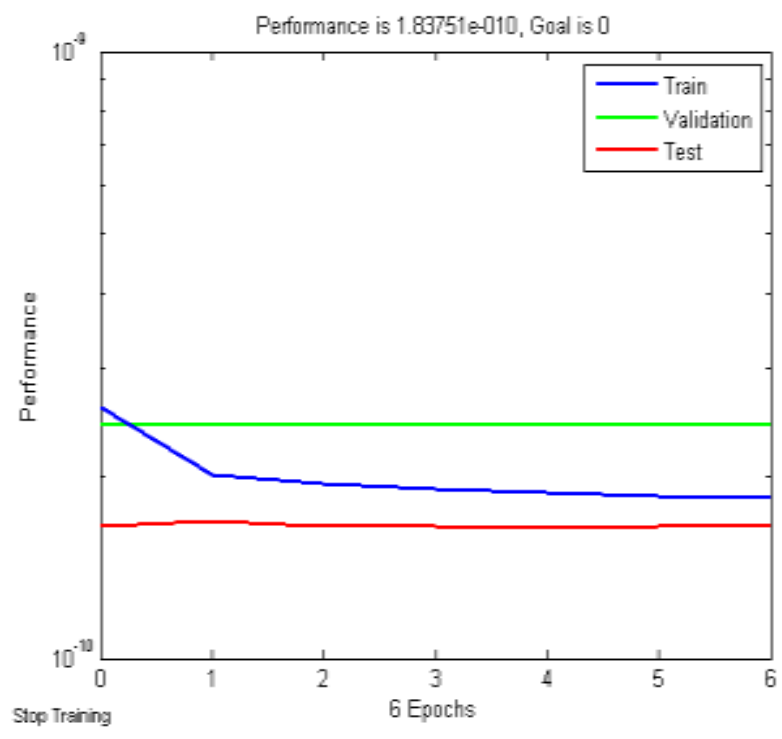

Fig.3 Performance behavior of the identification plant process
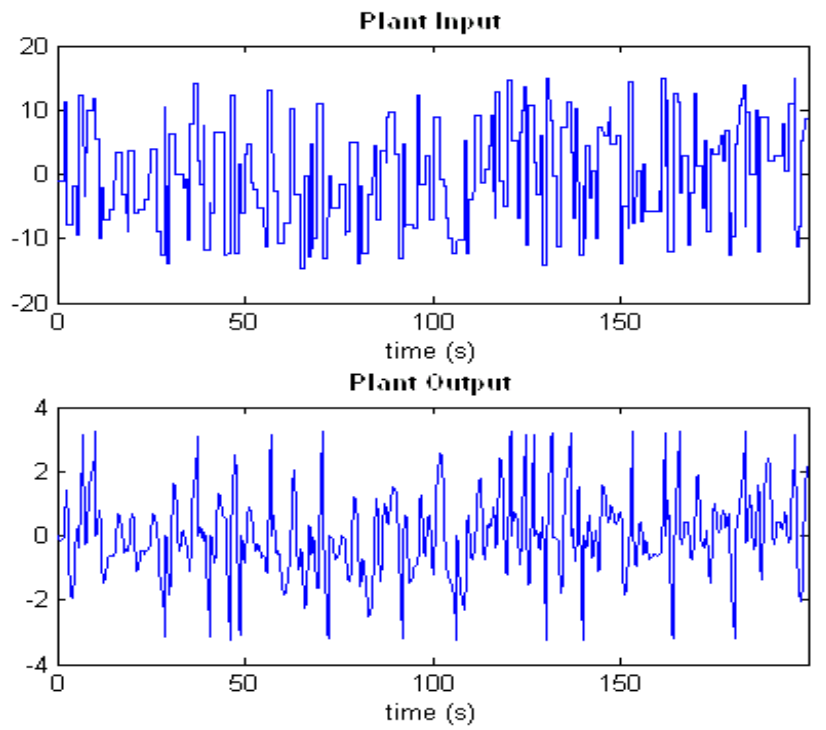

Fig.4 Input-output data taken during the plant identification process 
Khalil: Neural Network Based on Model Reference Using for Robot Arm -----
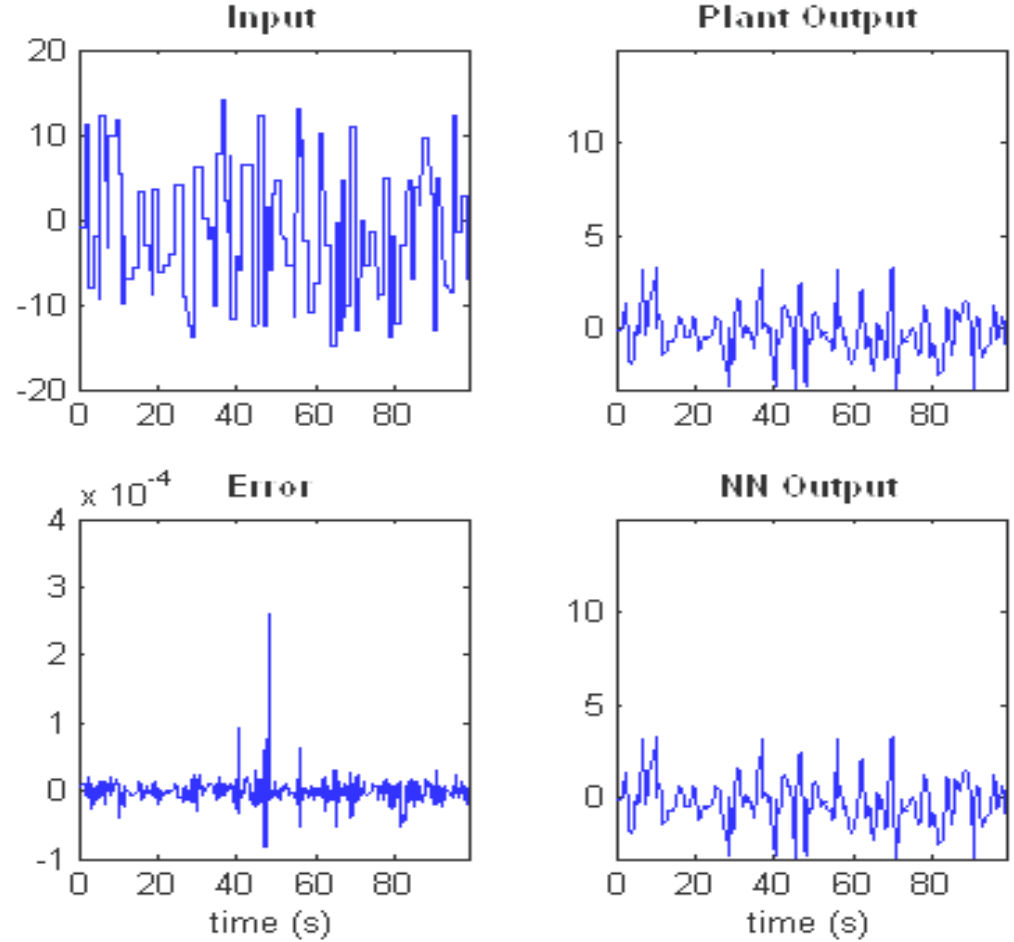

(a)
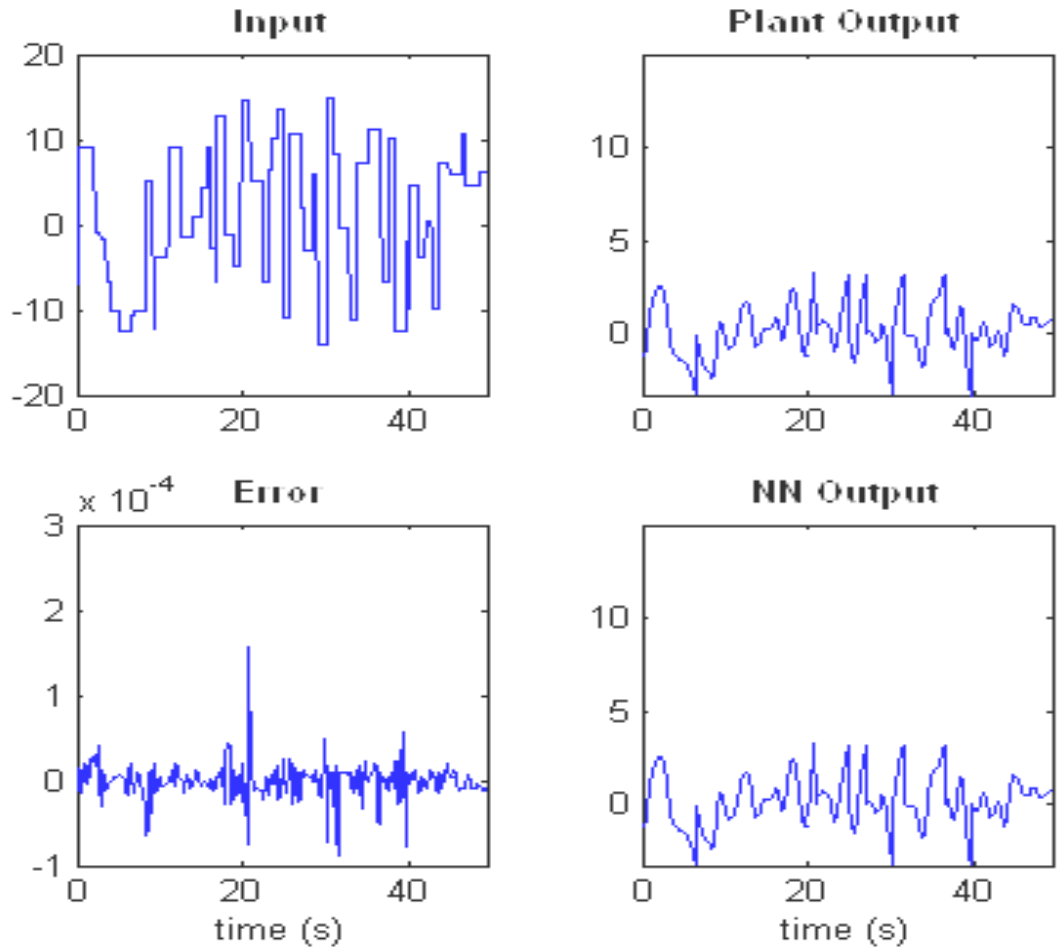

(b) 
No. 4

May 2014
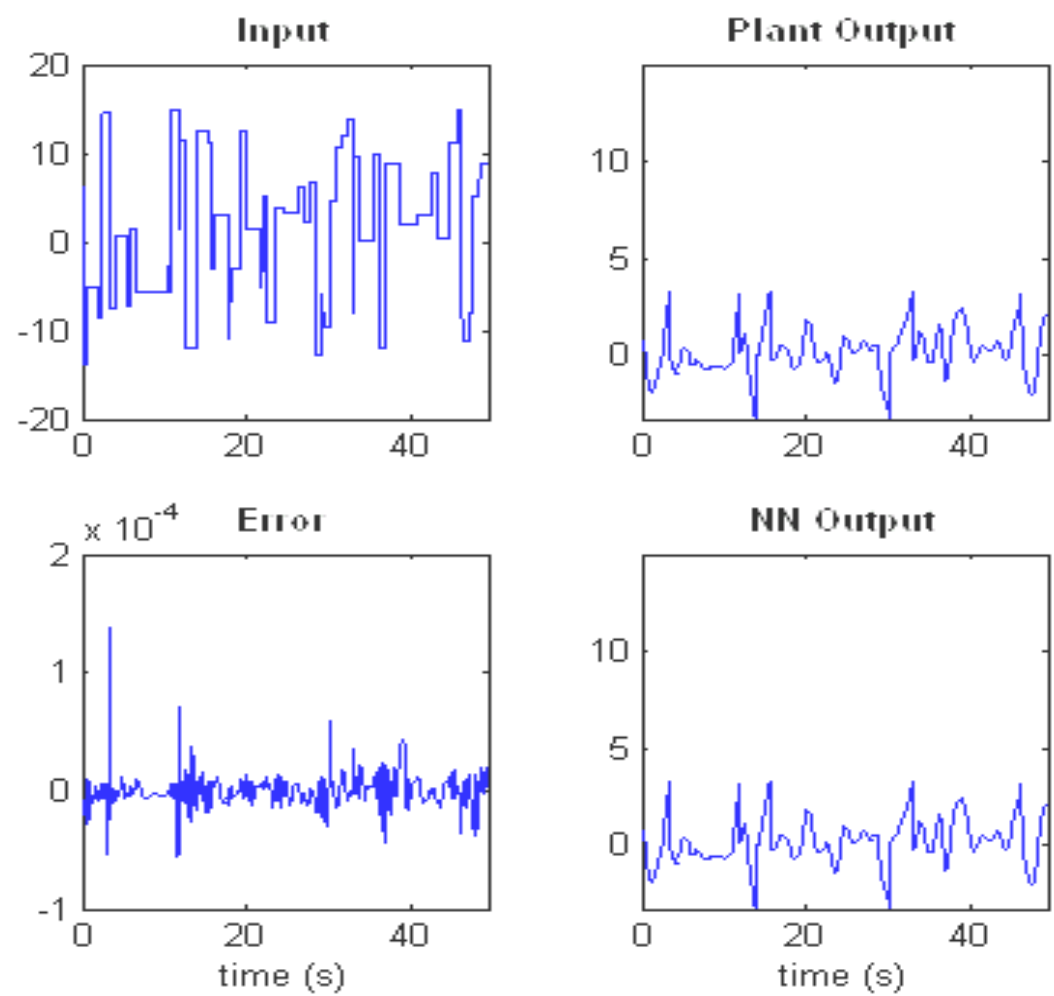

(c)

Fig.5 (a) Training data for model reference controller (b) results for reference model validation, and (c) test results.
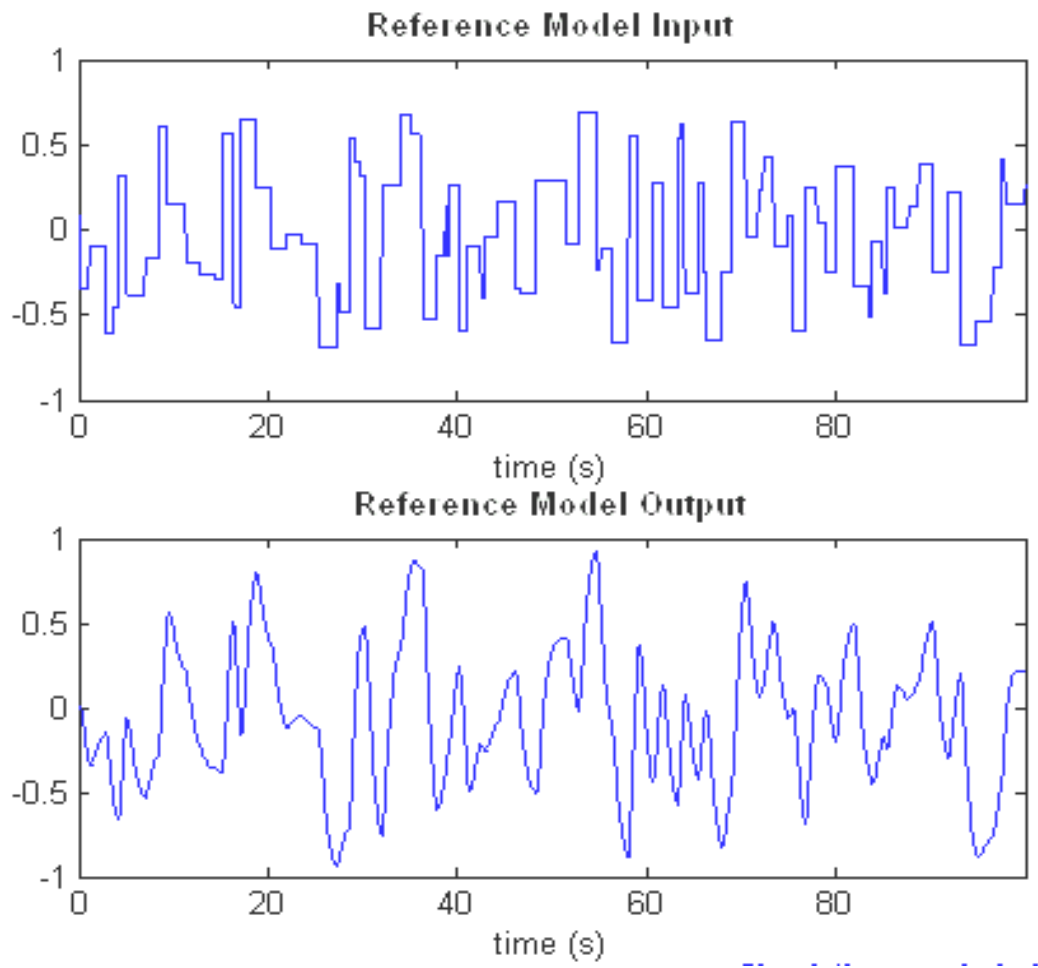

Fig.6 Input-output data for NN model reference controller 


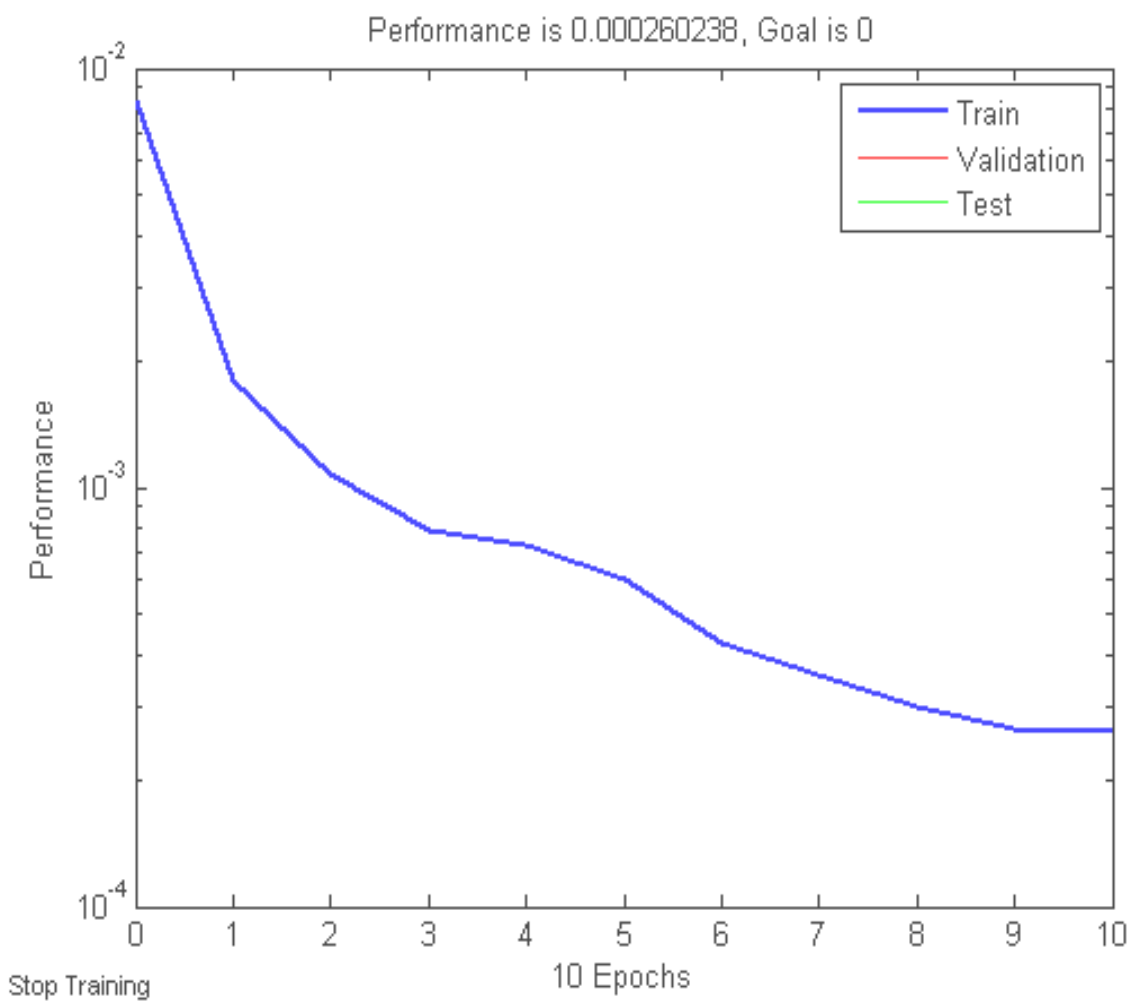

Fig.7 Controller training performance index behavior using trainbfgc.

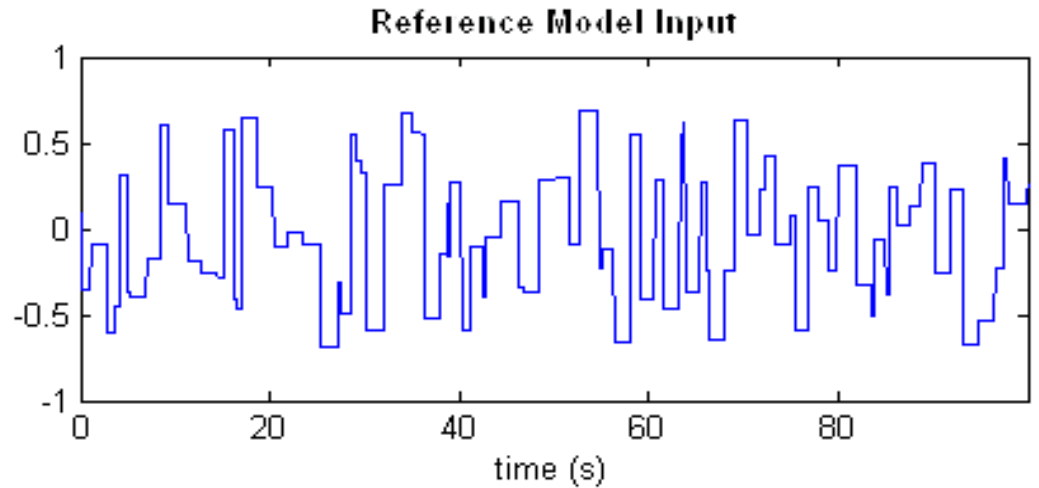

Reference Irodel output dbluek. Neural Network output dgleen\}

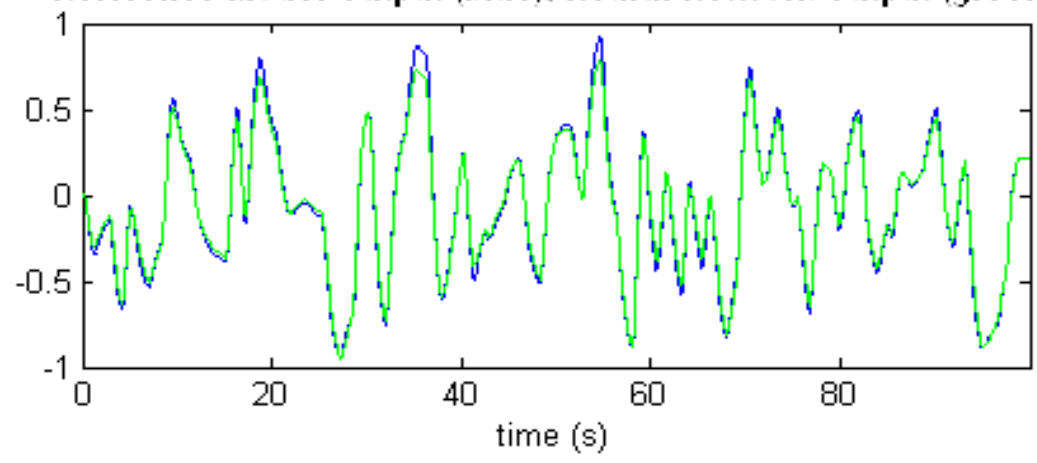

Fig.8 Dynamic responses of the reference and NN models 
No. 4

May 2014

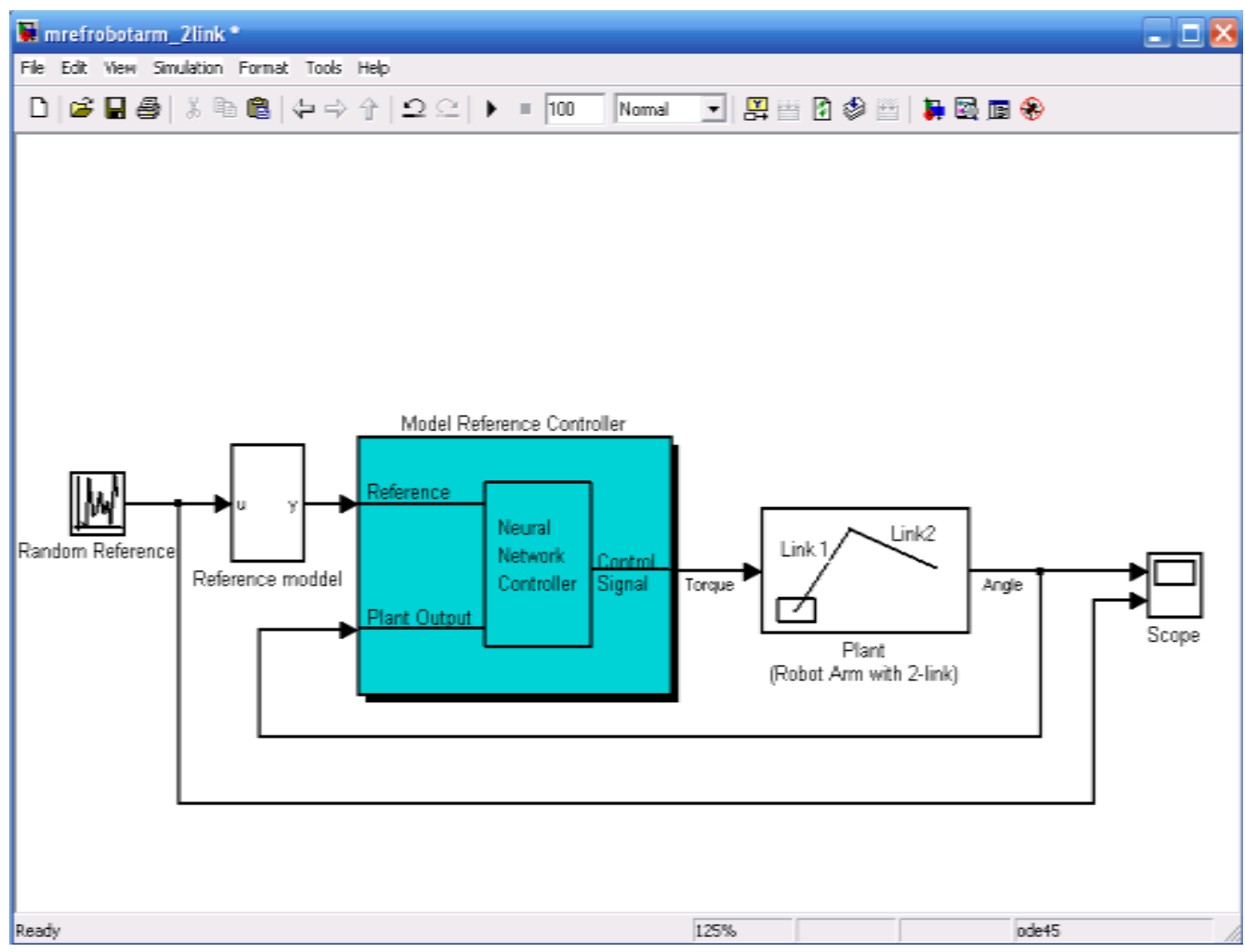

Fig.9 Robot arm modeling by Matlab-simulink.

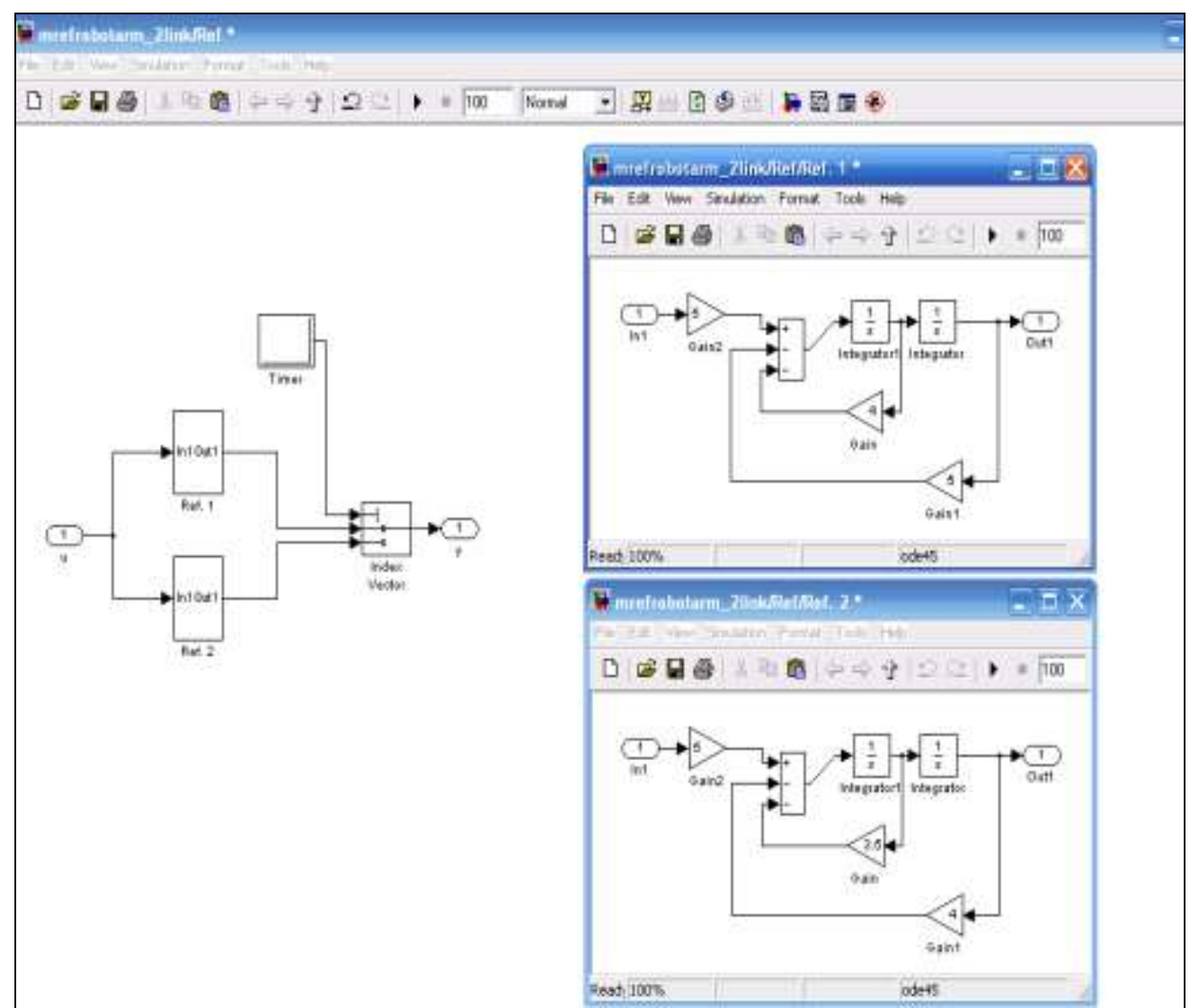

(a) 


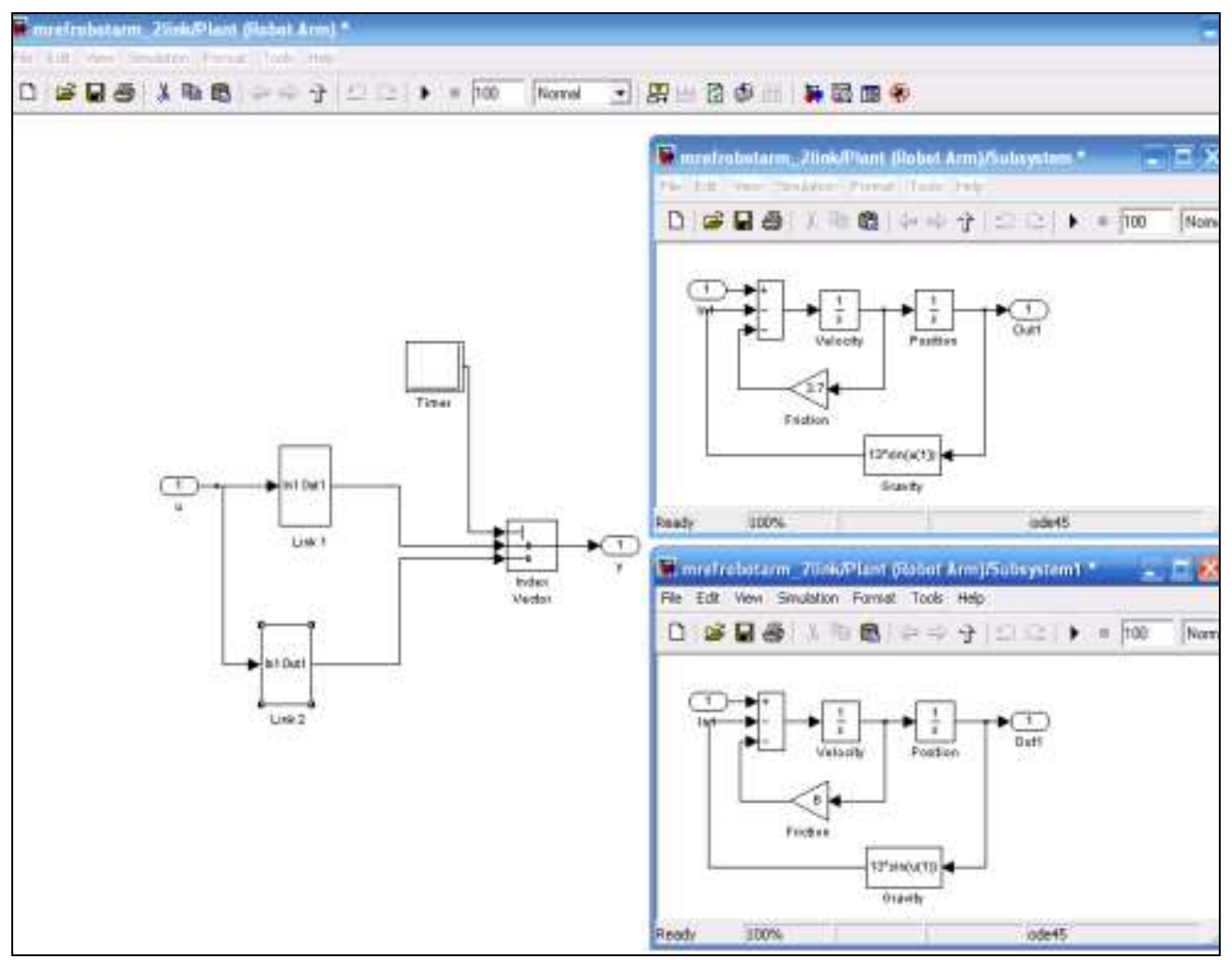

(b)

Fig.10 Block diagrams of the (a) reference model and (b) plant model.

\section{Simulation Results}

As shown in Fig.9, a random input reference is applied to the system and the results displayed on Fig.11. The dynamic behavior of both plant and the reference models is closed.

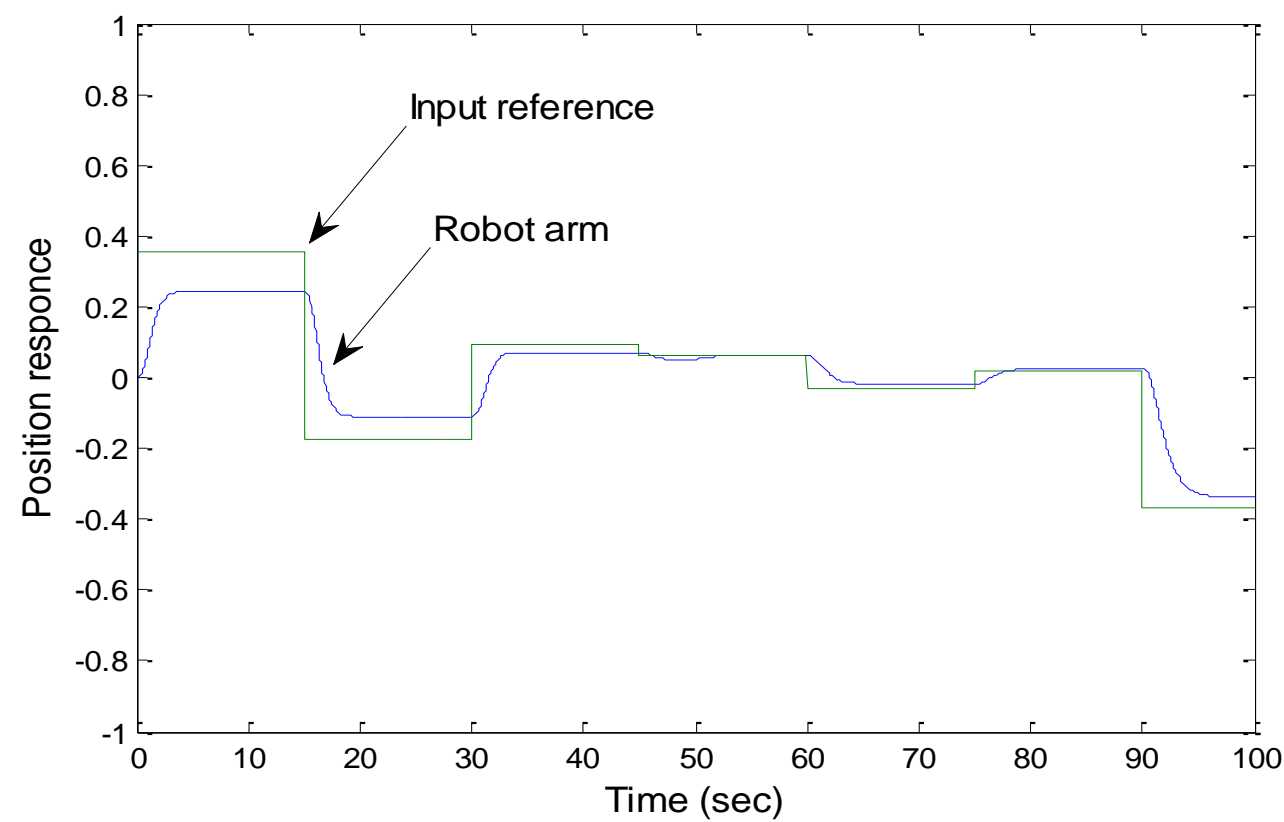

Fig.11 The robot arm and input reference responses 


\section{Conclusions}

In this paper, the NN controller based on reference model has been achieved. From the simulation results, the neural networks reproduce a model output from a plant. The adequate joint behavior of the overall system will depend on factors like model selection and its dynamics should be close to the plant, which want to control.

\section{References}

1. S. A. Abdulkereem and A. A. Ali, "Identification and Control of Three-Links Electrically Driven Robot Arm Using Fuzzy Neural Networks", International Journal of Robotics and Automation (IJRA), Vol. 2, Issue 1, pages:14-26, 2011.

2. J. Giergiel and K. Kurc, "Identification of the Mathematical Model of an Inspection Mobile Robot with Fuzzy Logic Systems and Neural Networks", Journal of Theoretical and Applied Mechanics, pages: 209-225, Warsaw, 2011.

3. M. Kalyoncu and M. Tinkir, "Mathematical Model for Simulation and Control of Nonlinear Vibration of A Single Flexible Link", 5th International Symposium on Intelligent Manufacturing Systems, pages:435-442, Sakarya University, Turkey, 2006.

4. M. Mailahi and J. C. Wue Shiung, "control of A Robot Arm using Iterative Learning Algorithm with A Stopping Criterion", Journal Technology, page: 55-72, University Technology, Malaysia, 2002.

5. C. R. Marcano-Gamero, "Plant Identification and Control using A Neural Network Based on Reference Model", Electronics Engineering, University Nacional, Experimental Politécnica, Puerto Ordaz - Venezuela, 2004.

6. G. Simmons and Y. Demiris, "Optimal Robot Arm Control using The Minimum Variance Model", Journal of Robotic Systems, Department of Electrical and Electronic Engineering, Imperial College, London, 2005.

7. P. Melin and O. Castillo, "Soft Computing for Intelligent Control of Nonlinear Dynamical Systems", International Journal of Computational Cognition, Vol. 2, No.1, pages: 45-78, March 2004.

The work was carried out at the college of Engineering. University of Mosul 